\title{
Clinical application of heart rate variability after acute myocardial infarction
}

\author{
Heikki V. Huikuri ${ }^{1}$ and Phyllis K. Stein ${ }^{2}$ \\ ${ }^{1}$ Division of Cardiology, Department of Internal Medicine, Institute of Clinical Medicine, University of Oulu, Oulu, Finland \\ ${ }^{2}$ Washington University School of Medicine, St. Louis, MO, USA
}

Edited by:

Federico Lombardi, University of

Milan, Italy

Reviewed by:

Marko S. Laaksonen, Mid Sweden

University, Sweden

Sergey V. Nesterov, University of

Turku, Finland

*Correspondence:

Heikki V. Huikuri, Department of

Internal Medicine, Institute of Clinical

Medicine, University of Oulu, P.O. Box

5000 (Kajaanintie 50), FIN-90014

Oulu, Finland.

e-mail: heikki.huikuri@oulu.fi
Heart rate (HR) variability has been extensively studied in patients surviving an acute myocardial infarction (AMI). The majority of studies have shown that patients with reduced or abnormal HR variability/turbulence have an increased risk of mortality within few years after an AMI. Various measures of HR dynamics, such as time-domain, spectral, and non-linear measures of HR variability, as well as HR turbulence, have been used in risk stratification of post-AMI patients. The prognostic power of various measures, except of those reflecting rapid $R-R$ interval oscillations, has been almost identical, albeit some non-linear HR variability measures, such as short-term fractal scaling exponent, and HR turbulence, have provided somewhat better prognostic information than the others. Abnormal HR variability predicts both sudden and non-sudden cardiac death after AMI. Because of remodeling of the arrhythmia substrate after AMI, early measurement of HR variability to identify those at high risk should likely be repeated later in order to assess the risk of fatal arrhythmia events. Future randomized trials using HR variability/turbulence as one of the pre-defined inclusion criteria will show whether routine measurement of HR variability/turbulence will become a routine clinical tool for risk stratification of post-AMI patients.

Keywords: mortality, coronary artery disease, sudden cardiac death
The purpose of this mini-review is to discuss the role of measurement of heart rate (HR) variability in patients after an acute myocardial infarction (AMI), especially its potential or proven clinical utility, by itself or in combination with other variables. Studies will be reviewed that employed a large variety of HR variability measures, with the majority derived from easily obtainable 24-h recordings.

Clinical utility is, of course, a broad concept. In this review, we will consider a measure to have clinical utility, if it identifies patients at higher risk of an adverse outcome, differentiates those with more advanced from those with less advanced disease, identifies those who would benefit from interventions or identifies those who are benefiting from or being harmed by an intervention. HR variability has been applied in a huge number of studies. We have chosen to limit our review to main studies focusing on clinical application of HR variability measurements from 24-h Holter recordings in patients who have survived an AMI.

\section{BACKGROUND FOR ABNORMAL HR VARIABILITY}

Heart rate variability is a term that encompasses a large number of measures of different types that have been described in details in other articles of this Special Topic of the journal. Timedomain measures reflect "how much" HRV there is. In general, if such measures are extremely low, it can be assumed that there is true autonomic dysfunction, but higher values for these various measures could reflect more healthy autonomic function or an unhealthy, highly irregular HR pattern (erratic rhythm). Frequency domain measures have the same properties. Extremely low values are associated with a lack of autonomic modulation of HR, but higher values, without examination of their underlying organization using power spectral plots or other graphical methods, cannot be assumed to reflect better HR variability. Nonlinear measures like the short-term fractal scaling exponent and the power-law slope, appear to better differentiate between healthy and unhealthy organization of the heart's rhythm and have proved to be more sensitive for risk stratification that some of the standard measures (Bigger et al., 1996; Huikuri et al., 2000). HR turbulence, which was covered recently in detail (Bauer et al., 2008), also appears to be sensitive to autonomic dysfunction, especially to impaired baroreflex function. It is likely that the optimal characterization of normal and abnormal HR variability would be based on a combination of these different HR variability parameters rather than on a single one.

\section{PROGNOSTIC SIGNIFICANCE OF HR VARIABILITY AFTER AMI}

The prognostic significance of $\mathrm{HR}$ variability has been extensively studied in patients who have survived an AMI. Table 1 summarizes the results of main studies assessing the prognostic significance of HR variability measurements from 24 -h recordings. As early as Wolf et al. (1978) showed that patients with a low magnitude of short-term HR variability (no sinus arrhythmia on 30 consecutive R-R intervals on admitting ECG) had a poor prognosis after AMI. In the late 1980s, the multicenter post-infarction project (MPIP) confirmed the predictive value of reduced HRV by measuring 24-h SD of N-N intervals (SDNN) over $24 \mathrm{~h}$ following AMI (Kleiger et al., 1987). Adjustment for covariates did not explain 
Table 1 | Summary of main studies assessing the prognostic significance of heart rate variability after acute myocardial infarction.

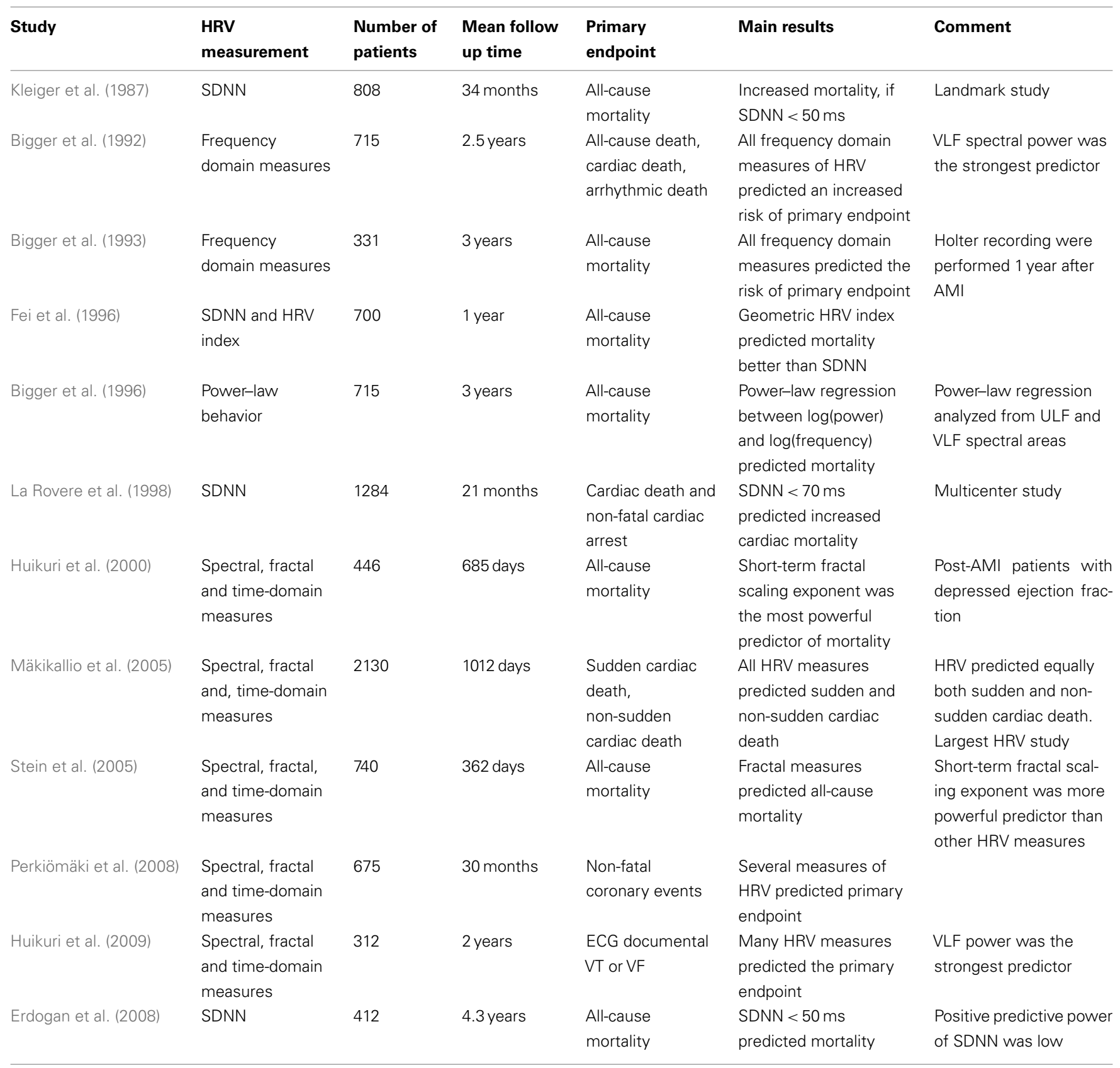

AMI, acute myocardial infarction; ECG, electrocardiogram; HRV, heart rate variability; SDNN, SD of N-N intervals; ULF, ultra-low frequency; VF, ventricular fibrillation; $V L F$, very-low frequency; VT, ventricular tachycardia.

this association. Other studies (including a reanalysis of MPIP) have shown that spectral measures of HR variability, mainly the very-low and low frequency spectral components, are reduced in survivors of AMI and that decreased values are associated with an increased risk of all-cause mortality (Bigger et al., 1992).

Because accurate Holter scanning is relatively labor intensive, the group at St. George's hospital in London experimented with global, geometric indices of $\mathrm{HR}$ variability (HR variability index) that were derived from the histogram of $\mathrm{N}-\mathrm{N}$ intervals (Malik et al., 1989). Decreased values for these measures were also found to be associated with higher risk of mortality (Fei et al., 1996). In addition, this group suggested that markedly reduced SDNN for a single, stable 5-min period could pre-select patients in whom 24-h Holter recordings using geometric methods would provide significant risk stratification.

The autonomic tone and reflexes after myocardial infarction (ATRAMI) trial was a multicenter observational study performed about 10 years after MPIP. The ATRAMI investigators confirmed 
that reduced $24-\mathrm{h}$ SDNN ( $<70 \mathrm{~ms}$ in this case) is associated with an increased mortality during 21 months of follow up (La Rovere et al., 1998). Furthermore, the combination of low SDNN and left ventricular ejection fraction $<35 \%$ carried a high risk of mortality.

Early studies focused on measurement of HR variability relatively early after AMI. Measures of HR variability appeared to be more depressed at the early phase of AMI with substantial improvement during recovery (Jokinen et al., 2003). Despite this autonomic recovery, measurement of $\mathrm{HR}$ variability has been shown to yield similar prognostic information when the 24-h HRV measurements were performed late after AMI (Bigger et al., 1993; Jokinen et al., 2003).

The post-MI studies performed in the 1980s and 1990s used 24$\mathrm{h}$ time and frequency domain as well as geometric measures of HR variability for risk stratification of patients after AMI. Although all statistical, geometrical, and spectral measures of HR variability differ in their manner of computation and analysis, these methods are fundamentally based on moment statistics and describe the magnitude of HR variability or of its underlying components. It is therefore not surprising that most of these measures that have been shown to have prognostic value have relatively close mutual correlation, and that there are only minor differences in prognostic power between them. It must be kept in mind though that measures of beat-to-beat HR variability which are supposed to reflect respiratory related vagal control of HR (HF power, pNN50, rMSSD) have rarely shown a strong association with outcome. This is likely because of the high prevalence of erratic rhythm in these populations resulting in higher measures for these parameters that do not reflect better parasympathetic function. One study showed that when erratic short-term R-R intervals was excluded from the analysis, HR variability related to respiratory cycles predicted sudden cardiac death even better than the standard HR variability indexes (Peltola et al., 2008).

More recent studies have applied methods based on HR turbulence, non-linear dynamic, and maximum deceleration capacity of $\mathrm{R}-\mathrm{R}$ intervals, which provide very different and perhaps complementary information on HR dynamics compared to traditional statistical methods (Mäkikallio et al., 2002; Bauer et al., 2006, 2008). Methods based on non-linear dynamics and HR turbulence have provided somewhat better prognostic information than that obtained by traditional methods (Bauer et al., 2006, 2008; Perkiömäki et al., 2008). In particular, decreased short-term fractal scaling exponent by the DFA method (called DFA1 or $\alpha 1$ ), a measure of greater randomness in HR patterns, has turned out to be a powerful non-linear index in risk stratification, a more powerful prognostic tool than other HR variability indexes in post-AMI populations (Huikuri et al., 2000; Mäkikallio et al., 2002). In the DIAMOND-MI trial, reduced short-term fractal scaling exponent identified post-AMI patients at a relative high risk of mortality and was more strongly related to outcome than traditional time and frequency domain measures (Huikuri et al., 2000). Moreover, reduced scaling exponent was related to both arrhythmic and non-arrhythmic mortality (Huikuri et al., 2000). In another study of consecutive patients with acute MI, both reduced short-term fractal scaling exponent and power-law slope were independently associated with recurrent non-fatal coronary events (Perkiömäki et al., 2008). Another measure of increased randomness of HR patterns, such as the ratio of the axes of a Poincare plot fitted to the plot of each $\mathrm{N}-\mathrm{N}$ interval vs. the next, also predicted mortality in the CAST trial (Stein et al., 2005). Patients in the CAST were at variable times post-MI but were selected for having significant ventricular arrhythmias.

One study in the era of modern therapy showed a very-low incidence of severely depressed HR variability (SDNN $<50 \mathrm{~ms}$; Erdogan et al., 2008). All patients in the study were treated with direct percutaneous coronary angioplasty within $12 \mathrm{~h}$ of their event. Although the 4-year survival was significantly higher in the low SDNN group, the positive predictive value was low, suggesting that the predictive power of HR variability may not be as high as earlier in patients receiving optimal medical and revascularization therapy. However, results were based on traditional time-domain HR variability measures and there was no report of results for non-linear measures or HR turbulence in this population.

\section{HR VARIABILITY AS A PREDICTOR OF ARRHYTHMIC EVENTS/SUDDEN CARDIAC DEATH}

The studies assessing the prognostic power of HR variability after AMI have usually used all-cause mortality as the primary endpoint (see Table 1). Some studies, using various definitions of arrhythmic events in their designs, have suggested that reduced or altered HR variability may be specifically related to arrhythmic events and sudden cardiac death. However, the clinical applicability of measurement of HR variability as a predictor of fatal arrhythmic events was challenged in a randomized prophylactic implantable cardioverter-defibrillator (ICD) trial, the defibrillator in AMI trial (DYNAMIT) which, however, used reduced SDNN combined with reduced left ventricular ejection fraction measured early (within 2 weeks) after AMI as an inclusion criterion for the trial. The trial could not show any mortality benefit from ICD therapy in these presumably high risk patients (Hohnloser et al., 2004). The more recent multicenter cardiac arrhythmias and risk stratification after myocardial infarction (CARISMA) study showed that reduced HR variability measured relatively late (6 weeks after AMI) rather than early after AMI, especially the very-low frequency spectral component, was the most powerful index among many other non-invasive risk markers in predicting the fatal or near-fatal arrhythmic events diagnosed by implantable arrhythmia devices (Huikuri et al., 2009). A further sub analysis of the CARISMA and another recent similar study (Risk estimation after infarction, non-invasive evaluation, REFINE) study confirmed that HR variability and HR turbulence yield more powerful prognostic information for arrhythmic events when measured late (6-10 weeks after AMI) rather than early (within 2 weeks) after AMI in the current treatment era (Huikuri et al., 2010). Thus the lack of recovery from reduced autonomic function after AMI was a stronger predictor of adverse events than decreased autonomic function early after AMI. A large study including more than 2000 post-AMI patients (FINGER-study) showed that reduced HR variability and HR turbulence predicted sudden cardiac death better among those with a left ventricular ejection fraction $>40 \%$ than in those with ejection fraction $<40 \%$ (Mäkikallio et al., 2005). 


\section{HR VARIABILITY AS A PREDICTOR OF NON-SUDDEN CARDIAC DEATH}

Observational studies using various end-points have shown that reduced HR variability also predicts non-sudden cardiac death. The largest study in this field showed that reduced HR variability/turbulence is in fact a stronger predictor of non-sudden than sudden cardiac death (Mäkikallio et al., 2005). In this study, the majority of patients were treated according to current guidelines, i.e., with primary coronary interventions, beta-blocking medication, and angiotensin converting enzyme inhibitors.

Considered together, the available data show that abnormal HR variability, measured by various time and frequency domain measures, non-linear measures, and turbulence/deceleration capacity measures from $24-\mathrm{h} \mathrm{R}-\mathrm{R}$ interval recordings is a general risk marker for common modes of cardiac death: arrhythmic, vascular, and hemodynamic after AMI. HR variability/turbulence measured early after AMI seems to provide more powerful information on the risk of early non-sudden cardiac death, especially due to progressive heart failure. Because of remodeling of the arrhythmia substrate after AMI, early measurement of HR variability to identify those at high risk of early mortality should likely be repeated later in order to assess the true risk of fatal or near-fatal arrhythmia events.

Often forgotten, however, in this focus on risk stratification, is the negative predictive value of HR variability measures. Patients with normal HR variability measures, even if they are post-MI, are at very-low risk of mortality. However, as has been found with other non-invasive risk markers, the positive predictive accuracy and sensitivity of abnormal HR variability for adverse outcomes has remained low in most observational studies. The sensitivity, specificity, and predictive values of HR variability in predicting future events depend partly on defining the cutoff points of abnormal HR variability measures. The cutoff points vary between the studies and depend on the study populations, such as post-AMI patients with and without heart failure, usage of beta-blocking medication etc., which influence the HR variability measures, and there is no universal consensus on the ideal cutoffs for different measures. Age and presence of diabetes and even clinical depression are other factors influencing HR variability and should be considered in defining the cutoff of abnormal measures. HR variability has been combined with other risk markers in order to improve risk stratification in some studies. The problem with combining various risk markers, however, is the overall loss of sensitivity despite an improved positive predictive accuracy.

\section{EFFECT OF INTERVENTIONS ON HR VARIABILITY}

Finally, little is known about the effect of interventions on HR variability in post-AMI patients and about whether interventions that are associated with improvements in HR variability are associated with better outcomes or vice versa. One intervention, coronary artery bypass surgery $(\mathrm{CABG})$ is associated

\section{REFERENCES}

Bauer, A., Kantelhardt, J. W., Barthel, P., Schneider, R., Mäkikallio, T. H., Ulm, K., Hnatkova, K., Schöning, A., Huikuri, H. V., Bunde, A., Malik, M., and Schmidt, G. (2006).
Deceleration capacity of heart rate as a predictor of mortality after myocardial infarction: cohort study. Lancet 367, 1674-1681.

Bauer, A., Malik, M., Schmidt, G., Barthel, P., Bonnemeier, H.,

with markedly decreased HR variability which slowly improves over weeks and months (Stein et al., 2004). It is likely that any predictive value for long-term outcomes contained in traditional time and frequency domain HR variability is lost once the patient has undergone CABG surgery. However, two studies have shown that measurements of non-linear HRV parameters after CABG surgery did identify those at higher risk of a complicated post-surgical course (Laitio et al., 2000; de Godoy et al., 2009). Only one trial, the BHAT (Beta-Blocker Heart Attack Trial) examined change in HR variability with therapy and the subsequent relationship of $\mathrm{HR}$ variability to mortality (Lampert et al., 2003). In BHAT, patients randomized to propranolol had a significantly greater increase in HF power at 6-weeks and after 21 months of follow up, higher HR variability at 6 weeks and assignment to the beta-blocker arm were independent predictors of a combined endpoint. On the other hand, anti-arrhythmic therapies that proved to be associated with reduced survival have been associated with negative effects on HR variability. In a meta-analysis of the effect of pharmacologic, behavioral, and exercise strategies on HR variability, Nolan et al. (2008) concluded that these secondary preventive strategies have a moderate but significant positive effect on HR variability. However, none of the large-scale studies on post-MI patients conducted so far have shown that improvement of HR variability by any of the therapeutic interventions would be associated with better outcome.

\section{FUTURE CHALLENGES AND RECOMMENDATIONS}

Future research in this field should focus on randomized trials that take into consideration the timing of HR variability/turbulence measurement after AMI in their study designs. Information from such trials may identify the populations in which the clinical applicability of routine measurement of HR variability is warranted. Measurement of the evolution of HR variability over time in association with prescribed therapies, perhaps on an individual basis, may also add to the clinical value of HR variability. Measures of HR variability should be combined with other clinical risk variables or biomarkers, in future studies to create risk scoring for post-AMI patients based on multiple risk factors. Currently, ongoing study (REFINE-ICD), where patients with reduced HR turbulence, abnormal time-domain T-wave alternans, and left ventricular ejection fraction between 35 and $50 \%$ measured 2-14 months after AMI are being randomized to ICD therapy (ICD) and standard therapy (personal communication). If mortality benefit is observed in the ICD arm of this trial, measurement of HR turbulence will become a routine clinical tool for a large number of post-AMI patients. Before the completion of the trial or other similar trials, patients with low HR variability/turbulence after AMI should receive the best available medical and invasive therapy, rehabilitation, and follow-up, even if routine measurements of HR variability/turbulence cannot yet be recommended in clinical practice guidelines.

Cygankiewicz, I., Guzik, P., Lombardi, F., Müller, A., Oto, A., Schneider, R., Watanabe, M., Wichterle, D., and Zareba, W. J. (2008). Heart rate turbulence: standards of measurement,

\begin{abstract}
physiological interpretation, and clinical use: International Society for Holter and Noninvasive Electrophysiology Consensus. J. Am. Coll. Cardiol. 52, 1353-1365.
\end{abstract}


Bigger, J. T. Jr., Fleiss, J. L., Rolnitzky, L. M., and Steinman, R. C. (1993). Frequency domain measures of heart period variability to assess risk of late myocardial infarction. J. Am. Coll. Cardiol. 21, 729-736.

Bigger, J. T. Jr., Fleiss, J. L., Steinman, R. C., Rolnitzky, L. M., Kleiger, R. E., and Rottman, J. N. (1992). Frequency domain measures of heart period variability and mortality after myocardial infarction. Circulation $85,164-171$

Bigger, J. T. Jr., Steinman, R. C., Rolnitzky, L. M., Fleiss, J. L., Albrecht, P., and Cohen, R. J. (1996). Power law behavior of RR-interval variability in healthy middle-aged persons, patients with recent acute myocardial infarction and patients with heart transplants. Circulation 93, 2142-2151.

de Godoy, M. F., Takakura, I. T., Correa, P. R., Machado, M. N., Miranda, R. C., and Brandi, A. C. (2009). Preoperative nonlinear behavior in heart rate variability predicts morbidity and mortality after coronary artery bypass graft surgery. Med. Sci. Monit. 15, CR117-CR122.

Erdogan, A., Coch, M., Bilgin, M., Parahuleva, M., Tillmanns, H., Waldecker, B., and Soydan, N. (2008). Prognostic value of heart rate variability after acute myocardial infarction in the era of immediate reperfusion. Herzschrittmacherther. Elektrophysiol. 19, 161-168.

Fei, L., Copie, X. X., Malik, M., and Camm, A. J. (1996). Short- and longterm assessment of heart rate variability for risk stratification after acute myocardial infarction. Am. J. Cardiol. 77, 681-684.

Hohnloser, S. H., Kuck, K. H., Dorian, P., Roberts, R. S., Hampton, J. R., Hatala, R., Fain, E., Gent, M., Connolly, S. J., and DINAMIT Investigators. (2004). Prophylactic use of an implantable cardioverterdefibrillator after acute myocardial infarction. N. Engl. J. Med. 351, 2481-2488.

Huikuri, H. V., Exner, D. V., Kavanagh, K. M., Aggarwal, S. G., Mitchell, L. B., Messier, M. D., Becker, D.,
Sheldon, R. S., Bloch Thomsen, P. E., CARISMA, and REFINE Investigators. (2010). Attenuated recovery of heart rate turbulence early after myocardial infarction identifies patients at high risk of fatal or near-fatal arrhythmic events. Heart Rhythm 7, 229-235.

Huikuri, H. V., Mäkikallio, T. H., Peng, C.-K., Goldberger, A. L., Hintze, U., Møller, M., and the Diamond Study Group. (2000). Fractal correlation properties of R-R interval dynamics and mortality in patients with depressed left ventricular function after an acute myocardial infarction. Circulation 101, 47-53.

Huikuri, H. V., Raatikainen, M. J. P., Moerch-Joergensen, R., Hartikainen, J., Virtanen, V., Boland, J., Anttonen, O., Hoest, N., Boersma, L. V. A., Platou, E. S., Messier, M. D., BlochThomsen, P.-E., and for the Cardiac Arrhythmias and Risk Stratification after Acute Myocardial Infarction (CARISMA) study group. (2009). Prediction of fatal or near fatal cardiac arrhythmia events in patients with depressed left ventricular function after an acute myocardial infarction. Results of the Cardiac Arrhythmias and Risk Stratification after Acute Myocardial Infarction (CARISMA) Study. Eur. Heart J. 30, 689-698.

Jokinen, V., Tapanainen, J. M., Seppänen, T., and Huikuri, H. V. (2003). Temporal changes and prognostic significance of measures of heart rate dynamics after acute myocardial infarction in the beta-blocking era. Am. J. Cardiol. 92, 907-912.

Kleiger, R. E, Miller, J. P., Bigger, J. T., Moss, A. J., and the Multicenter Post-infarction Research Group. (1987). Decreased heart rate variability and its association with increased mortality after myocardial infarction. Am. J. Cardiol. 59, 256-262.

La Rovere, M. T., Bigger, J. T. Jr., Marcus, F. I., Mortara, A., Schwartz, P. J., and ATRAMI (Autonomic Tone and Reflexes After Myocardial Infarction) Investigators. (1998). Baroreflex sensitivity and heart-rate variability in prediction of total cardiac mortality after myocardial infarction. Lancet 351, 478-484.

Laitio, T. T., Huikuri, H. V., Kentala, E. S., Mäkikallio, T. H., Jalonen, J. R., Helenius, H., Sariola-Heinonen, K., YliMäyry, S., and Scheinin, H. (2000). Correlation properties and complexity of perioperative RR-interval dynamics in coronary artery bypass surgery patients. Anesthesiology 93 69-80.

Lampert, R., Ickovics, J. R., Viscoli, C. J., Horwitz, R. I., and Lee, F. A (2003). Effects of propranolol on recovery of heart rate variability following acute myocardial infarction and relation to outcome in the BetaBlocker Heart Attack Trial. Am. J. Cardiol. 91, 137-142.

Mäkikallio, T. H., Barthel, P., Schneider, R., Bauer, A., Tapanainen, J. M., Tulppo, M. P., Schmidt, G. and Huikuri, H. V. (2005). Prediction of sudden cardiac death after acute myocardial infarction; role of Holter monitoring in the modern treatment era. Eur. Heart J. 26, 762-769.

Mäkikallio, T. H., Tapanainen, J. M., Tulppo, M. P., and Huikuri, H. V. (2002). Clinical applicability of heart rate variability analysis by methods based on nonlinear dynamics. Card. Electrophysiol. Rev 6, 250-255.

Malik, M., Farrell, T., Cripps, T., and Camm, A. J. (1989). Heart rate variability in relation to prognosis after myocardial infarction: selection of optimal processing techniques. Eur. Heart J. 10, 1060-1074.

Nolan, R. P., Jong, P., Barry-Bianchi, S. M., Tanaka, T. H., and Floras, J. S. (2008). Effects of drug, biobehavioral and exercise therapies on heart rate variability in coronary artery disease: a systematic review. Eur. J. Cardiovasc. Prev. Rehabil. 15, 386-339.

Peltola, M., Tulppo, M. P., Kiviniemi, A., Hautala, A. J., Seppänen, T., Barthel, P., Bauer, A., Schmidt, G. Huikuri, H. V., and Mäkikallio, T. H. (2008). Respiratory sinus arrhythmia as a predictor of sudden cardiac death after myocardial infarction. Ann. Med. 40, 376-382.
Perkiömäki, J. S., Jokinen, V., Tapanainen, J., Airaksinen, K. E., and Huikuri, H. V. (2008). Autonomic markers as predictors of nonfatal acute coronary events after myocardial infarction. Ann. Noninvasive Electrocardiol. 13, 120-129.

Stein, P. K., Domitrovich, P. P., Huikuri, H. V., Kleiger, R. E., and Cast Investigators. (2005). Traditional and nonlinear heart rate variability are each independently associated with mortality after myocardial infarction. J. Cardiovasc. Electrophysiol. 16 13-20.

Stein, P. K., Domitrovich, P. P., Kleiger, R. E., and CAST Investigators. (2004). Including patients with diabetes mellitus or coronary artery bypass grafting decreases the association between heart rate variability and mortality after myocardial infarction. Am. Heart J. 147, 309-316.

Wolf, M. M., Vargios, G. A., Hunt, D., and Sloman, J. G. (1978) Sinus arrhythmia in acute myocardial infarction. Med. J. Aust. 2, 52-53.

Conflict of Interest Statement: The authors declare that the research was conducted in the absence of any commercial or financial relationships that could be construed as a potential conflict of interest.

Received: 21 December 2011; paper pending published: 09 January 2012; accepted: 13 February 2012; published online: 27 February 2012.

Citation: Huikuri HV and Stein PK (2012) Clinical application of heart rate variability after acute myocardial infarction. Front. Physio. 3:41. doi: 10.3389/fphys.2012.0004

This article was submitted to Frontiers in Clinical and Translational Physiology, a specialty of Frontiers in Physiology.

Copyright (c) 2012 Huikuri and Stein. This is an open-access article distributed under the terms of the Creative Commons Attribution Non Commercial License, which permits non-commercial use, distribution, and reproduction in other forums, provided the original authors and source are credited. 